\title{
Rational Design of a Porous Supramolecular Peptide Framework (SPF): A Crystalline Tetraproline in the Polyproline II Conformation
}

\author{
Dominic F. Brightwell, Giada Truccolo, Kushal Samanta, Elliott J. Fenn, Simon J. Holder, Helena \\ J. Shepherd, and Aniello Palma*
}

Supramolecular Interfacial and Synthetic Chemistry Group, School of Physical Sciences, Ingram Building, University of Kent, Canterbury, CT2 $7 \mathrm{NH}, \mathrm{UK}$.

Supramolecular organic framework, short helical peptide, polyproline crystal structure, hydrogen bonded network

\begin{abstract}
Herein, we present the first high resolution single crystal structure of an unfunctionalized tetrameric proline in the polyproline II conformation. This rationally designed oligoproline tetramer, self-assembles to form a permanently porous crystalline supramolecular peptide framework (SPF). Thermal activation, guest inclusion and thermally induced release of chemical guests have been demonstrated for this novel system. This discovery provides a conclusive insight into the previously ambiguous conformation of short oligoprolines and will allow for the further development of proline-based peptide linkers in the rational design of SPFs and metal-peptide frameworks.
\end{abstract}

Peptide based porous frameworks are an emerging class of materials, which have found applications as adaptive ${ }^{1-3}$ and reversibly tuneable ${ }^{4}$ porous materials, capable of capturing greenhouse gases, 5,6 facilitating chiral events and transformations, ${ }^{7}$ and separating chiral drugs ${ }^{7,8}$. Peptides can be prepared at scale with high purity, have canonical and non-canonical amino acids incorporated into their primary sequence with high accuracy and have a high level of biocompatibility. As such, the efforts to investigate this class of compounds as an alternative to the rigid, aromatic and non-adaptable ligands in the creation of extended frameworks, has seen a surge in recent years..$^{7,9-13}$ While considerable advancement has been achieved in the field of peptide-based metal-organic frameworks (MOFs), $, 7,1,10,12,14,15$ it remains a challenge to develop peptidebased extended porous networks guided by supramolecular interactions (e.g. H-bonding, halogen bonding, $\pi$-interactions and host-guest interactions). ${ }^{16-18}$ To the best of our knowledge, the use of cyclic peptides to yield porous channels in crystalline materials and the use of self-assembling hydrophobic dipeptides, are amongst the few examples of well characterised peptide-based frameworks (i.e. Supramolecular Peptide Frameworks; SPFs). 5,13,19,20 In recognition of the immense chemical space yet to be explored in this field, we set out to exploit peptides with stable secondary structures in the construction of SPFs. In particular, we focused our efforts on the use of polyproline helices as SPF building blocks due to the accessibility to rigid yet stimuli sensitive secondary structures. ${ }^{21}$ Polyprolines can interconvert between two different secondary conformations, polyproline II with all trans amide bonds and polyproline I with all cis amide bonds. This interconversion can be controlled as a function of the environment it is exposed to (i.e. temperature, solvent polarity and $\mathrm{pH}){ }^{22-24}$ Polyproline helices have shown remarkable resilience to a diverse range of functionalisation and, in contrast to other helices used in supramolecular chemistry, can retain their conformation even in exceptionally short and un-functionalised sequences. ${ }^{25}$ These properties make them promising candidates for the synthesis of novel porous SPFs. Herein, we report the first crystal structure of the unfunctionalized tetrameric oligoproline $\mathbf{P P}_{4}$ in the polyproline II form (Figure 1).

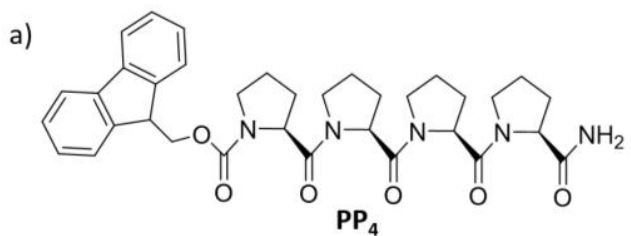

b)
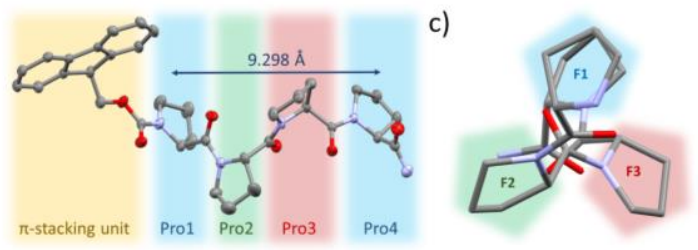

Figure 1. a) Proline tetramer Fmoc-(Pro $)_{4}-\mathrm{NH}_{2}\left(\mathbf{P P}_{4}\right)$, b) Crystal structure of $\mathbf{P P}_{4}, 50 \%$ ellipsoids (Mercury), c) View along the axis displaying $\mathrm{C}_{3}$ symmetry and the 3 faces $\left(\mathrm{F}_{1}-3\right)$ of the helix, Fmoc group removed for clarity. 
a)

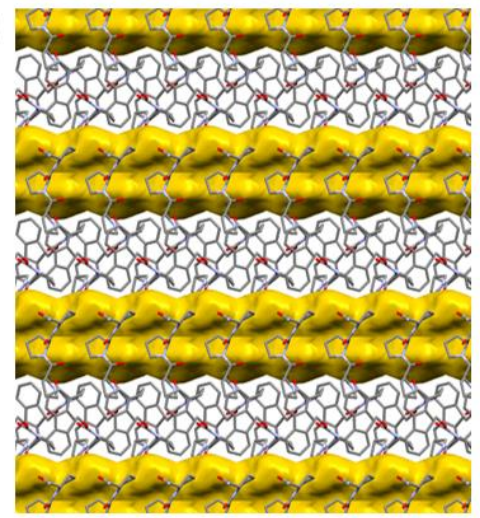

d)

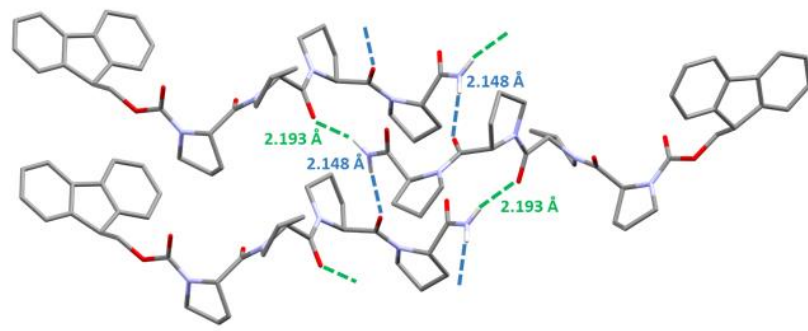

b)

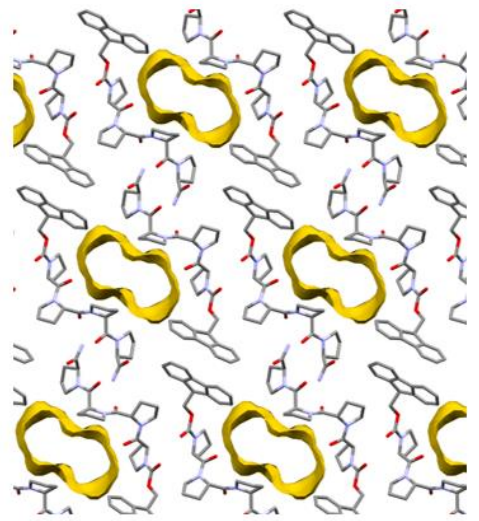

c)

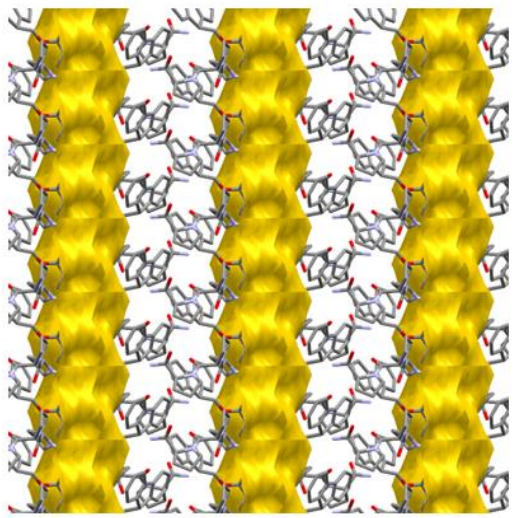

e)

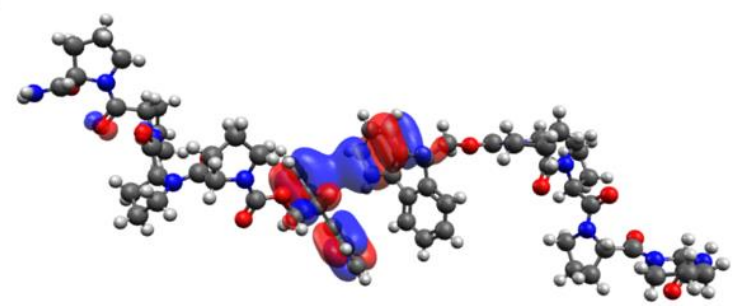

Figure 2. a) Crystal structure of $\mathbf{P P}_{4}$ showing packing and solvent accessible voids (yellow) viewed along the $a$ axis, ethanol molecules were removed, b) View along the $b$ axis, c) View along the $c$ axis, d) Hydrogen bonding between a single peptide unit and two opposing peptide units, through terminal amide hydrogens and Proz and Pro3 carbonyls (2.1930(18) Å and 2.1478(19) Å respectively), which extends through the framework, e) Model of PP4 dimer illustrating orbital interactions between Fmoc groups.

To the best of our knowledge, this is the shortest crystalline unfunctionalized polyproline II helix reported. ${ }^{26,27}$ This peptide was rationally designed to yield a porous supramolecular peptidic framework via hydrogen bonding and $\pi$ interactions. This remarkably resilient SPF is stable to thermal activation and can reversibly host inorganic molecules $\left(\mathrm{I}_{2}\right)$ within the channels while retaining its original supramolecular hosting network.

As the shortest oligoproline showing evidence of a polyproline II form in solution is a tetramer, ${ }^{25}$ we decided to focus our initial efforts on this type of oligomer. The earliest crystal structure of a tetrameric oligoproline was reported by Matsuzaki, with several of the proline units deviating from the typical polyproline II conformation. ${ }^{28}$ It was not until 2014, that Wennemer's group reported the first crystal structure of a polyproline hexamer in the polyproline II conformation providing insight into the stability of the polyproline helix, ${ }^{26}$ followed by Hanessian, who reported the crystal structure of the tetrameric proline congener (cis-4,5-methanoproline) in the polyproline II form. However, as stated in this work, the capacity for unfunctionalized tetrameric oligoprolines to adopt a polyproline II helical conformation was not conclusive. ${ }^{27}$

Analysis of the single crystal data in the literature for polyproline helices, allowed us to gain insight into the type of supramolecular interactions governing assembly. For example; in the case of the hexamer a hydrogen bond interaction between the lone pair on the carbonyl oxygen of the second proline and the $\mathrm{C}$ terminus carboxylic acid $(\mathrm{H}$ --O distance of 2.05(7) A) was present. ${ }^{26}$ With this insight in hand, we anticipated that a terminal primary amide could act as an excellent H-Donor $\left(\mathbf{H}_{\mathbf{D}}\right)$, which may promote the formation of an extended network through $\mathrm{H}$ bonds with $\mathrm{H}$-Acceptor $\left(\mathbf{H}_{\boldsymbol{A}}\right)$ groups (i.e. carbonyl groups). The $\mathrm{N}$ terminus was protected with a Fmoc carbamate group so as to facilitate intramolecular assembly of peptides via $\pi$ interactions. ${ }^{29,30}$ Moreover, analysing the hexamer crystal structure, we observed that the polyproline units packed in parallel with minimal void space. ${ }^{26}$ We hypothesised that the steric demand associated with the Fmoc functional group might force two helices into an antiparallel arrangement, hence promoting intermolecular $\pi$ interactions. With these design principles in mind, $\mathbf{P P}_{\mathbf{4}}$ was successfully synthesised using solid phase peptide methodology on a $0.3 \mathrm{mmol}$ scale and in a quantitative yield (SI 1). $\mathbf{P P}_{4}$ was characterised in solution with far-UV circular dichroism (CD) analysis of aqueous, ethanol, and propanol solutions ( $0.25 \mathrm{mM})$, showing that the peptide adopts a polyproline II helix conformation in all solvents $\left(\lambda_{\max }\right.$ at 223/229 $\mathrm{nm}$ and $\lambda_{\min }$ at $202 \mathrm{~nm}$; SI 5, Figure S6). High resolution mass spectrometry of $\mathbf{P P}_{4}$ evidenced the presence of dimers in solution $\left(2 \mathrm{M}+\mathrm{H}^{+}\right)$, suggesting that the supramolecular $\mathbf{H}_{D} / \mathbf{H}_{\boldsymbol{A}}$ interactions are present not just in the solid state (SI 2, Figure $\mathrm{S}_{2}$ ).

Robust crystals of $\mathbf{P P}_{4}$ suitable for single crystal X-ray diffraction analysis were reproducibly obtained via slow cooling of a super-saturated hot solution of $\mathbf{P P}_{\mathbf{4}}$ in ethanol (SI 7). The structure was solved and refined to an atomic resolution of $0.81 \AA$ (Figure 2), revealing that the peptide adopts the polyproline II helical conformation. This is the shortest unfunctionalized oligoproline reported to adopt 
this conformation in the solid state. The characteristic dihedral angles and an analysis of the puckering of the pyrrolidine rings, have been summarised in Table $S_{1}$ (SI 9.2). Detailed investigation of the helical structure of the peptide from crystal structure data revealed aspects in agreement with observations from the hexameric structure; we found that pyramidalization of the amide carbonyls clearly indicated the presence of $n-\pi^{*}$ interactions and these interactions were more significant in prolines exhibiting the exo conformation with a larger degree of pyramidalization (SI 9.3, Table 2). ${ }^{26}$ Analysis of the packing of $\mathbf{P P}_{4}$ showed an extended supramolecular peptide framework $\left(\mathbf{P P}_{4}-\mathbf{S P F}\right)$ with channels extending in one dimension through the network, which are occupied with disordered solvent molecules (SI 9, Figure S8).

As per our design, the formation of the extended framework is driven by a combination of hydrogen bonding and $\pi$-interactions. The peptides were arranged in alternating anti-parallel rows, extending through the network, such that each protected $\mathrm{N}$ terminus faces another Fmoc group (Figure 2e). Each row of peptides is offset from the next row such that the C-terminal amide can hydrogen bond with two other peptides. In particular, the amide group of each peptide interacts with different carbonyl groups on the two opposing peptides. The C-terminal amides hydrogen bond with the carbonyl group of Proz on peptide 1 and the Pro3 carbonyl on peptide 2 (Figure 2d, 2.1478(19) Å and 2.1930(18) A respectively), therefore each peptide acts as both a $\mathbf{H}_{D}$ and $\mathbf{H}_{A}$ with another peptide, which then extends in one dimension. These layers are then stacked in an alternating manner creating an extended network.

The crystal structure suggests that interactions between the Fmoc groups determine the second aspect of the selfassembly process. The closest distance (2.7515(19) Å) between the Fmoc moieties is that of a proton of one fluorenyl to the aromatic region of another (SI 9, Figure 11), well within the possible distance to classify a potential interaction. ${ }^{29,31,32}$ Computational calculations at [B3LYP$\left.\mathrm{D}_{3}(\mathrm{BJ}) / 6-311++\mathrm{G}(\mathrm{d}, \mathrm{p})\right]$ on a Fmoc associated pair (geometry fixed to that of the crystal structure) showed orbital overlap between the groups (Figure 2e and Sisz Figure 21). The electrostatic potential map showed relatively electron poor inner regions for the Fmoc groups, as expected, but no obvious points of significant electrostatic interaction between the groups (SI 13.1, Figure 20).

It is also worth noting that a reversable single crystal to single crystal transition in the unit cell of $\mathbf{P P}_{4}-\mathbf{S P F}$ (SI 9.19.3, $\left.\mathbf{P P}_{4}-\mathbf{S P F}_{\text {Flash }}\right)$ is observed, whereby the Pro3 puckering switches from exo to endo (SI 9.3, Table 1). This was achieved by flash freezing crystals at $150 \mathrm{~K}$. Remarkably, upon returning to room temperature the crystals readopt their initial unit cell and conformation. This was not found when slowly ramping the temperature down to $150 \mathrm{~K}$, with only a slight reduction of the cell volume, but no significant conformational changes.

Energy decomposition analysis (EDA) of the isolated dimer system (geometry fixed to that of the crystal structure) was used to determine the relative breakdown of the intermolecular forces between the Fmoc groups in the crystal lattice. Calculations were run with two different functionals, BLYP-D $3(B J)$ and PBE-D, giving interaction energies of -44.2 and $-32.4 \mathrm{kJmol}^{-1}$ respectively. The breakdown suggest that dispersion interactions are the predominant interaction between the Fmoc groups with smaller contributions from electrostatic and orbital interactions (and the Pauli repulsive interaction, SI 13.2, Table S3).

To investigate the ability for thermal release of ethanol from the framework channels, ${ }^{1} \mathrm{H}-\mathrm{NMR}$ analysis of $\mathbf{P P}_{4}-\mathbf{S P F}$ in methanol- $\mathrm{d}_{4}$ was carried out after activating the crystals for various times under vacuum at $45^{\circ} \mathrm{C}$. These studies revealed that thermal activation could gradually reduce the ethanol content over time up to a $90 \%$ reduction after 12 $\mathrm{h}$, compared to the initial content present within the framework (SI 10, Figure S15). Samples of $\mathbf{P P}_{4}$-SPF were ground before and after activation, and powder X-ray diffraction (PD-XRD) analyses were carried out with no reduction of crystallinity for activated samples compared to the original sample (SI 11, Figure S17). Simultaneous thermogravimetric analysis (TGA) and differential scanning calorimetry (DSC) of $\mathbf{P P}_{4}-\mathbf{S P F}$ showed a crystalline melting point with an endothermic peak $\left(150^{\circ} \mathrm{C}\right.$, SI 6, Figure $\left.\mathrm{S}_{7}\right)$.

To demonstrate the ability of $\mathbf{P} \mathbf{P}_{4}$-SPF to engage in hostguest chemistry, activated single crystals were exposed to a solution of iodine in hexane. By visual inspection of the crystals, a colour change was immediately evident with the crystals taking on a yellow appearance. a)

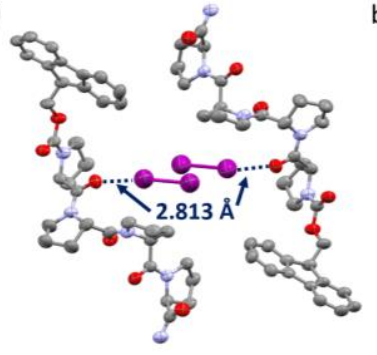

b)

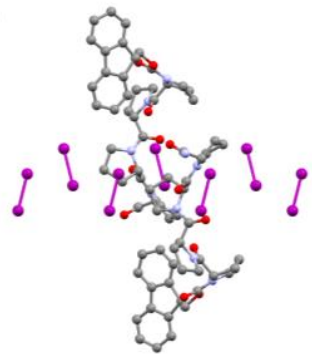

Figure 3. Crystal structures of $\mathbf{P P}_{4}-\mathbf{S P F} @ \mathbf{I}_{2}$, a) View along the b-axis, two parallel peptide units' halogen bond interactions (I---O distance of 2.813(16) $\AA$ ) with two molecules of iodine (purple), $50 \%$ ellipsoids (Mercury), b) View along the $a$ axis showing iodine filling the channels

SC-XRD analysis of $\mathbf{P P}_{4}-\mathbf{S P F} @ \mathbf{I}_{2}$ was performed which showed that the activated SPF was able to retain iodine with a chemical occupancy for $I_{2}$ of $\approx 12.3 \%$ (SI 12). The interatomic distances $(2.813(16) \AA$ ) between iodine and the Prol carbonyl oxygens were indicative of halogen bond formation (Figure 3a). To investigate the ability to perform reversible guest absorption, the same single crystal of $\mathbf{P P}_{4^{-}}$ SPF@ $\mathbf{I}_{2}$ was thermally treated, while mounted on the stage, using a heated flow of $\mathrm{N}_{2}$ at $50^{\circ} \mathrm{C}$ for 3 hours, prior to repeating SC-XRD analysis. The crystal was mounted without the use of any inert oil, to facilitate guest loss. Upon analysis of the thermally treated crystal, the chemical occupancy of $\mathrm{I}_{2}$ was reduced by $50 \%$ while crystallinity 
of the sample was retained, a clear indication of the stability of this SPF (SI 12). This experiment demonstrates the potential of $\mathbf{P P}_{4}-\mathbf{S P F}$ to perform thermally responsive guest release. Finally, a batch of $\mathbf{P P}_{\mathbf{4}}-\mathbf{S P F} @ \mathbf{I}_{2}$, kept at room temperature for four weeks, showed an $\mathrm{I}_{2}$ occupancy of 10 \% (Initial occupancy $12.3 \%$ ) from SC-XRD analysis, which exemplifies the necessity for thermal treatment to remove the guest $\mathrm{I}_{2}$.

In conclusion, the first crystal structure of an unfunctionalized tetrameric oligoproline in the polyproline II conformation is reported. The rational design of $\mathbf{P P} \mathbf{P}_{4}$ has resulted in the formation of the first polyproline based supramolecular peptide framework $\left(\mathbf{P P}_{4}-\mathbf{S P F}\right)$ which has shown remarkable stability and the ability to reversibly host chemical guests in its channels. The rational approach described herein to design $\mathbf{P P}_{\mathbf{4}}$-SPF will be applied to other peptidic systems with the aim to achieve cavity functionalisation and chemical separation (e.g. gasses and chiral mixtures), and will be reported in due course.

\section{ASSOCIATED CONTENT}

Synthetic methodology, NMR spectra, FT-IR, circular dichroism spectra, Mass spectrometry, X-ray diffraction data, simultaneous thermogravimetric analysis and computational data are available online in the Supporting Information.

CCDC-2127748-2127751 contain the supplementary crystallographic data for this paper, including structure factors and refinement instructions and can be obtained free of charge from The Cambridge Crystallographic Data Centre, 12 Union Road, Cambridge CB2 ${ }_{2} E Z$, UK (e-mail: deposit@ccdc.cam.ac.uk), or via https://www.ccdc.cam.ac.uk/getstructures

\section{AUTHOR INFORMATION}

\section{Corresponding Author}

*Aniello Palma - Supramolecular Interfacial and Synthetic Chemistry Group, School of Physical Sciences, Ingram Building, University of Kent, Canterbury, $\mathrm{CT}_{2} 7 \mathrm{NH}$, UK.

Email: a.palma@kent.ac.uk

\section{Authors}

Dominic F Brightwell - Supramolecular and Interfacial Chemistry, School of Physical Sciences, The University of Kent, Giles Ln, Canterbury CT2 7NZ, Kent, United Kingdom. Email: dfb6@kent.ac.uk

Giada Truccolo - Supramolecular and Interfacial Chemistry, School of Physical Sciences, The University of Kent, Giles Ln, Canterbury CT2 7NZ, Kent, United Kingdom.

Email: gt263@kent.ac.uk

Kushal Samanta - Supramolecular and Interfacial Chemistry, School of Physical Sciences, The University of Kent, Giles Ln, Canterbury CT2 7 NZ, Kent, United Kingdom.

Email: k.samanta@kent.ac.uk
Elliott J Fenn - Supramolecular and Interfacial Chemistry, School of Physical Sciences, The University of Kent, Giles Ln, Canterbury CT2 7 NZ, Kent, United Kingdom.

Email: ef336@kent.ac.uk

Simon J Holder - Supramolecular and Interfacial Chemistry, School of Physical Sciences, The University of Kent, Giles Ln, Canterbury CT2 2 NZ, Kent, United Kingdom.

Email: s.j.holder@kent.ac.uk

Helena J Shepherd - Supramolecular and Interfacial Chemistry, School of Physical Sciences, The University of Kent, Giles Ln, Canterbury CT2 7 NZ, Kent, United Kingdom.

Email: h.j.sheperd@kent.ac.uk

\section{Author Contributions}

Experiments were planned and executed by D.F.B., K.S, E.J.F and A.P. X-Ray diffraction data were collected and analysed by G.T, D.F.B and H.J.S. NMR, LC-MS were performed by D.F.B, K.S. and A.P. Circular dichroism and STA were performed by D.F.B. Computational calculations were performed by S.J.H. All authors contributed to data interpretation and writing of the manuscript. All authors have given approval to the final version of the manuscript.

\section{Notes}

The authors declare no competing financial interest.

\section{ACKNOWLEDGMENT}

A.P., K.S. acknowledge financial support from the EPSRC (Grant number EP/To16140/1). D.F.B. acknowledges support from the University of Kent (Vice Chancellor's Fellowship). G.T. acknowledges financial support from the Royal Society of Chemistry (Grant Number R19-2762), A.P. acknowledges financial support from the Royal Society of Chemistry (Grant E21-9299054940).

\section{ABBREVIATIONS}

SI, Supporting Information; CD, Circular Dichroism; STA, Simultaneous thermogravimetric analysis; SC-XRD, Single crystal X-ray diffraction; PD-XRD, Powder X-ray diffraction; DSC, Differential scanning calorimetry MOF, Metalorganic framework; SOF, Supramolecular organic framework; SPF, Supramolecular peptide framework.

\section{REFERENCES}

(1) Rabone, J.; Yue, Y.-F.; Chong, S. Y.; Stylianou, K. C.; Bacsa, J.; Bradshaw, D.; Darling, G. R.; Berry, N. G.; Khimyak, Y. Z.; Ganin, A. Y.; Wiper, P.; Claridge, J. B.; Rosseinsky, M. J. An Adaptable PeptideBased Porous Material. Science (80). 2010, 329 (5995), 1053-1057. https://doi.org/10.1126/SCIENCE.1190672.

(2) Katsoulidis, A. P.; Antypov, D.; Whitehead, G. F. S.; Carrington, E. J.; Adams, D. J.; Berry, N. G.; Darling, G. R.; Dyer, M. S.; Rosseinsky, M. J. Chemical Control of Structure and Guest Uptake by a Conformationally Mobile Porous Material. Nat. 20195657738 2019, 565 (7738), 213-217. https://doi.org/10.1038/s41586-018-0820-9.

(3) Katsoulidis, A. P.; Park, K. S.; Antypov, D.; Martí-Gastaldo, C.; Miller, G. J.; Warren, J. E.; Robertson, C. M.; Blanc, F.; Darling, G. R.; Berry, N. G.; Purton, J. A.; Adams, D. J.; Rosseinsky, M. J. GuestAdaptable and Water-Stable Peptide-Based Porous Materials by 
Imidazolate Side Chain Control. Angew. Chem. Int. Ed. Engl. 2014, 53 (1), 193. https://doi.org/10.1002/ANIE.201307074.

(4) Martí-Gastaldo, C.; Antypov, D.; Warren, J. E.; Briggs, M. E.; Chater, P. A.; Wiper, P. V.; Miller, G. J.; Khimyak, Y. Z.; Darling, G. R.; Berry, N. G.; Rosseinsky, M. J. Side-Chain Control of Porosity Closure in Single- and Multiple-Peptide-Based Porous Materials by Cooperative Folding. Nat. Chem. 201464 2014, 6 (4), 343-351. https://doi.org/10.1038/NCHEM.1871.

(5) Comotti, A.; Bracco, S.; Distefano, G.; Sozzani, P. Methane, Carbon Dioxide and Hydrogen Storage in Nanoporous Dipeptide-Based Materials. Chem. Commun. 2009, No. 3, 284-286. https://doi.org/10.1039/B820200A.

(6) Yadav, V. N.; Comotti, A.; Sozzani, P.; Bracco, S.; Bonge-Hansen, T.; Hennum, M.; Görbitz, C. H. Microporous Molecular Materials from Dipeptides Containing Non-Proteinogenic Residues. Angew. Chemie Int. Ed. 2015, $54 \quad$ (52), 15684-15688. https://doi.org/10.1002/ANIE.201507321.

(7) Navarro-Sánchez, J.; Argente-García, A. I.; Moliner-Martínez, Y.; Roca-Sanjuán, D.; Antypov, D.; Campíns-Falcó, P.; Rosseinsky, M. J.; Martí-Gastaldo, C. Peptide Metal-Organic Frameworks for Enantioselective Separation of Chiral Drugs. J. Am. Chem. Soc. 2017, 139 (12), 4294-4297. https://doi.org/10.1021/jacs.7boo28o.

(8) Saito, A.; Sawada, T.; Fujita, M. X-Ray Crystallographic Observation of Chiral Transformations within a Metal-Peptide Pore. Angew. Chemie Int. Ed. 2020, 59 (46), 20367-20370. https://doi.org/10.1002/anie.202007731.

(9) Saito, A.; Sawada, T.; Fujita, M. X-Ray Crystallographic Observation of Chiral Transformations within a Metal-Peptide Pore. Angew. Chemie Int. Ed. 2020, 59 (46), 20367-20370. https://doi.org/10.1002/anie.202007731.

(10) Schnitzer, T.; Paenurk, E.; Trapp, N.; Gershoni-Poranne, R.; Wennemers, H. Peptide-Metal Frameworks with Metal Strings Guided by Dispersion Interactions. J. Am. Chem. Soc. 2021, 143 (2), 644-648. https://doi.org/10.1021/jacs.oc11793.

(11) Martí-Gastaldo, D. C.; Warren, D. J. E.; Briggs, D. M. E.; Armstrong, D. J. A.; Thomas, P. K. M.; Rosseinsky, P. M. J. SpongeLike Behaviour in Isoreticular $\mathrm{Cu}(\mathrm{Gly}-\mathrm{His}-\mathrm{X})$ Peptide-Based Porous Materials. Chemistry 2015, $21 \quad$ (45), 16027. https://doi.org/10.1002/CHEM.201502098.

(12) Mantion, A.; Massüger, L.; Rabu, P.; Palivan, C.; McCusker, L. B.; Taubert, A. Metal-Peptide Frameworks (MPFs): "Bioinspired" Metal Organic Frameworks. J. Am. Chem. Soc. 20o8, 130 (8), 2517-2526. https://doi.org/10.1021/jao762588.

(13) Mehrparvar, S.; Wölper, C.; Gleiter, R.; Haberhauer, G. The

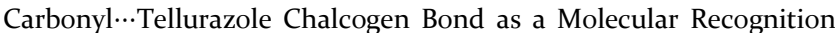
Unit: From Model Studies to Supramolecular Organic Frameworks. Angew. Chemie Int. Ed. 2020, 59 (39), 17154-17161. https://doi.org/10.1002/ANIE.202005374.

(14) Misra, R.; Saseendran, A.; Dey, S.; Gopi, H. N. Metal-Helix Frameworks from Short Hybrid Peptide Foldamers. Angew. Chemie 2019, 131 (8), 2273-2277. https://doi.org/10.1002/ange.201810849.

(15) Martí-Gastaldo, C.; Warren, J. E.; Stylianou, K. C.; Flack, N. L. O.; Rosseinsky, M. J. Enhanced Stability in Rigid Peptide-Based Porous Materials. Angew. Chemie Int. Ed. 2012, 51 (44), 11044-11048. https://doi.org/10.1002/ANIE.201203929.

(16) Li, P.; Ryder, M. R.; Stoddart, J. F. Hydrogen-Bonded Organic Frameworks: A Rising Class of Porous Molecular Materials. Accounts $\begin{array}{lllll}\text { Mater. } & \text { Res. } & \text { 2020, } & 1 & \text { (1), }\end{array}$ https://doi.org/10.1021/accountsmr.ocooo19.

(17) Yang, W.; Greenaway, A.; Lin, X.; Matsuda, R.; Blake, A. J.; Wilson, C.; Lewis, W.; Hubberstey, P.; Kitagawa, S.; Champness, N. R.; Schröder, M. Exceptional Thermal Stability in a Supramolecular Organic Framework: Porosity and Gas Storage. J. Am. Chem. Soc. 2o10, 132 (41), 14457-14469. https://doi.org/10.1021/ja1042935.
(18) Tian, J.; Chen, L.; Zhang, D. W.; Liu, Y.; Li, Z. T. Supramolecular Organic Frameworks: Engineering Periodicity in Water through HostGuest Chemistry. Chem. Commun. 2016, 52 (38), 6351-6362. https://doi.org/10.1039/c6cco2331b.

(19) Görbitz, C. H. Microporous Organic Materials from Hydrophobic Dipeptides. Chem. - A Eur. J. 2007, 13 (4), 1022-1031. https://doi.org/10.1002/CHEM.200601427.

(2o) Yadav, V. N.; Comotti, A.; Sozzani, P.; Bracco, S.; Bonge-Hansen, T.; Hennum, M.; Görbitz, C. H. Microporous Molecular Materials from Dipeptides Containing Non-Proteinogenic Residues. Angew. Chemie Int. Ed. 2015, $54 \quad$ (52), 15684-15688. https://doi.org/10.1002/ANIE.201507321.

(21) Aronoff, M. R.; Egli, J.; Schmitt, A.; Wennemers, H. Alkylation of $\gamma$-Azaproline Creates Conformationally Adaptable Proline Derivatives for PH-Responsive Collagen Triple Helices. Chem. - A Eur. J. 2020, 26 (22), 5070-5074. https://doi.org/10.1002/chem.201905768.

(22) RK, D.; TA, K. Reassessment of the Random Coil Conformation: Vibrational CD Study of Proline Oligopeptides and Related Polypeptides. Biopolymers 1991, $31 \quad$ (14), 1747-1761. https://doi.org/10.1002/BIP.360311409.

(23) El-Baba, T. J.; Fuller, D. R.; Hales, D. A.; Russell, D. H.; Clemmer, D. E. Solvent Mediation of Peptide Conformations: Polyproline Structures in Water, Methanol, Ethanol, and 1-Propanol as Determined by Ion Mobility Spectrometry-Mass Spectrometry. J. Am. Soc. Mass Spectrom. 2019, 30 (1), 77. https://doi.org/10.1007/S13361018-2034-7.

(24) Siebler, C.; Erdmann, R. S.; Wennemers, H. Switchable Proline Derivatives: Tuning the Conformational Stability of the Collagen Triple Helix by PH Changes. Angew. Chemie 2014, 126 (39), 1050810512. https://doi.org/https://doi.org/10.1002/ange.201404935.

(25) Kakinoki, S.; Hirano, Y.; Oka, M. On the Stability of PolyprolineI and II Structures of Proline Oligopeptides. Polym. Bull. 2005, 53 (2), 109-115. https://doi.org/10.1007/soo289-004-0317-6.

(26) Wilhelm, P.; Lewandowski, B.; Trapp, N.; Wennemers, H. A Crystal Structure of an Oligoproline PPII-Helix, at Last. J. Am. Chem. Soc. 2014, 136 (45), 15829-15832. https://doi.org/10.1021/ja507405j.

(27) Berger, G.; Vilchis-Reyes, M.; Hanessian, S. Structural Properties and Stereochemically Distinct Folding Preferences of 4,5 -Cis and Trans-Methano-L-Proline Oligomers: The Shortest Crystalline PPIIType Helical Proline-Derived Tetramer. Angew. Chemie Int. Ed. 2015, 54 (45), 13268-13272. https://doi.org/10.1002/anie.201506208.

(28) Matsuzaki, T. The Crystal Structure of T-ButyloxycarbonyltetraL-Proline Benzyl Ester. Acta Crystallogr. Sect. B Struct. Crystallogr. Cryst. Chem. 1974, 30 (4), 1029-1036. https://doi.org/10.1107/so567740874004213.

(29) McGaughey, G. B.; Gagné, M.; Rappé, A. K. $\pi$-Stacking Interactions: ALIVE AND WELL IN PROTEINS*. J. Biol. Chem. 1998, 273 (25), 15458-15463. https://doi.org/10.1074/jbc.273.25.15458.

(30) Eckes, K. M.; Mu, X.; Ruehle, M. A.; Ren, P.; Suggs, L. J. $\beta$ Sheets Not Required: Combined Experimental and Computational Studies of Self-Assembly and Gelation of the Ester-Containing Analogue of an Fmoc-Dipeptide Hydrogelator. Langmuir 2014, 30 (18), 5287-5296. https://doi.org/10.1021/LA500679B.

(31) Nishio, M. The $\mathrm{CH} / \pi$ Hydrogen Bond in Chemistry. Conformation, Supramolecules, Optical Resolution and Interactions Involving Carbohydrates. Phys. Chem. Chem. Phys. 2011, 13 (31), 138731390o. https://doi.org/10.1039/c1cp20404a.

(32) Sakaki, S.; Kato, K.; Miyazaki, T.; Musashi, Y.; Ohkubo, K.; Lhara, H.; Hirayama, C. Structures and Binding Energies of BenzeneMethane and Benzene-Benzene Complexes An A\& Inifio SCF/MPS Study. J. Am. Soc. Faraday Trans, 1993, 89 (4), 659-664. 
Insert Table of Contents artwork here

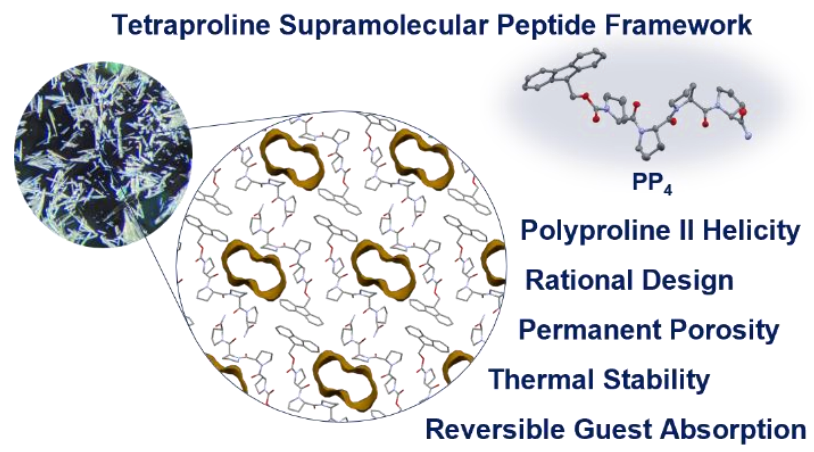

